\title{
Quest for the Missing Kidney in the "Treasure Chest": Report of a Thoracic Kidney in a Child With Recurrent Diaphragmatic Hernia
}

\author{
Ahmed Adam ${ }^{\mathrm{a},}{ }^{*}$, Mariza De Villiers ${ }^{\mathrm{b}}$, Gertruida Van Biljon $^{\mathrm{c}}$ \\ ${ }^{a}$ Department of Urology, University of Pretoria, Pretoria, South Africa \\ ${ }^{\mathrm{b}}$ Department of Paediatric Surgery, University of Pretoria, Pretoria, South Africa \\ ${ }^{c}$ Department of Paediatric Nephrology, University of Pretoria, Pretoria, South Africa
}

*Corresponding author: Department of Urology, University of Pretoria, Steve Biko Academic Hospital, Room 71207, Level 7, Bridge C, Private Bag X 169, Pretoria, Gauteng 0001 South Africa. E-mail: aadam81@gmail.com

\begin{abstract}
The thoracic cavity is the rarest location of all the renal ectopic sites. We report a rare case of an acquired thoracic kidney associated with a previous congenital diaphragmatic hernia in a child with nephrotic syndrome. Although only 13 cases of the thoracic kidney in the pediatric age group have been described in worldwide reports during the past 25 years, we present the first to be associated with a recurrent diaphragmatic hernia. The classification, differential diagnosis, and management options of this rare form of ectopia are discussed.
\end{abstract}

\section{Introduction}

The thoracic kidney is mostly asymptomatic and is, therefore, rarely found in childhood. ${ }^{1}$ We report the presence of an acquired thoracic kidney in a child with nephrotic syndrome and a history of a previous diaphragmatic hernia repair.

\section{Case report}

A 6-year-old boy presented to the pediatric outpatient department with a 2-week history of coughing and generalized edema. The surgical history was significant. He had undergone congenital diaphragmatic repair performed while he was a neonate, after he had presented with acute respiratory distress soon after birth. He had recovered well after the repair and had remained asymptomatic until the current presentation.

On clinical examination, abdominal ascites and bilateral stony dullness with marked decreased air entry on the left lower lung lobe were present. The urine dipstick test revealed a nephrotic range of proteinuria. Additional hematologic investigations showed mild azotemia, hypoalbuminemia, and hypercholesterolemia. The initial 
chest radiograph confirmed the presence of bilateral effusions and an obscured left hemidiaphragm with an abnormal cardiac silhouette.

These clinical findings were in keeping with a diagnosis of nephrotic syndrome, and medical treatment with oral steroid therapy was initiated.

During additional diagnostic workup, abdominal ultrasonography revealed ascites and an "absent" left kidney. Because of the previously reported abnormality of the cardiac silhouette, cardiac ultrasonography was also performed, excluding the presence of any underlying cardiac pathologic entity. The follow-up chest radiograph (Fig. 1) showed complete resolution of the pleural effusions, revealing the underlying left-sided posterior mediastinal mass.

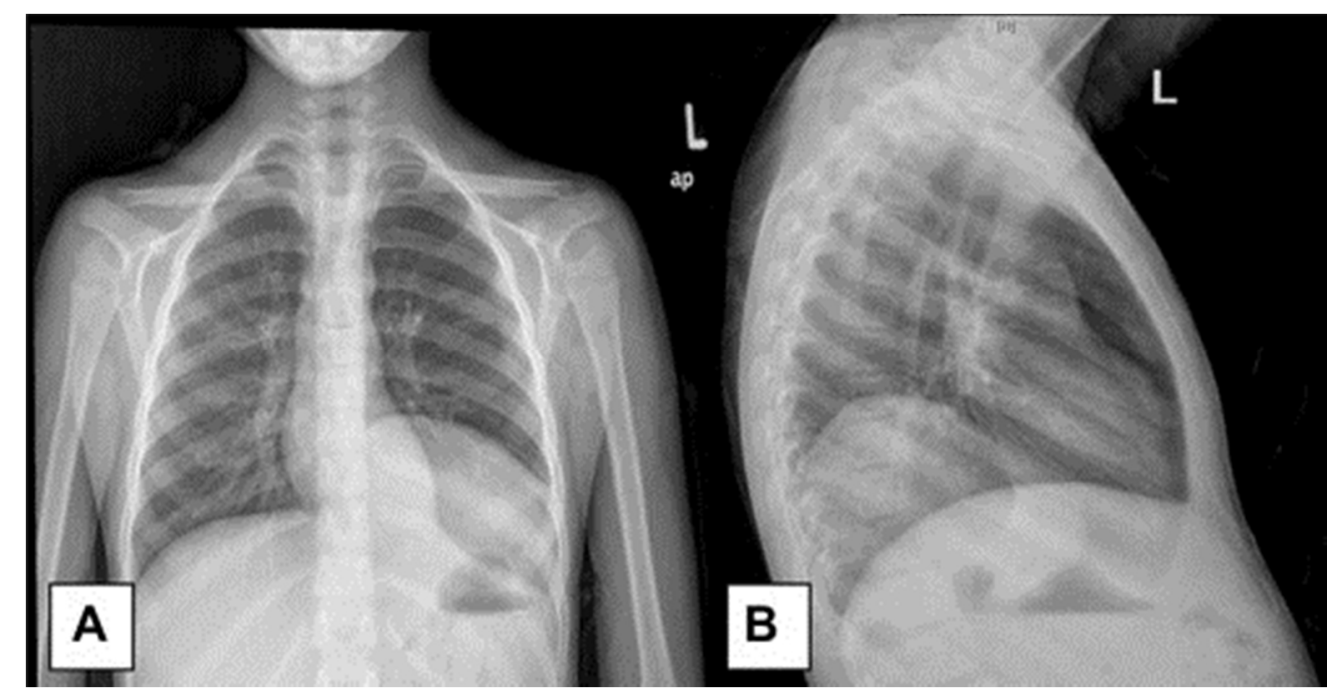

Figure 1. Chest radiograph ([A] anteroposterior view; $[\mathrm{B}]$ lateral view) revealing left-sided posterior mediastinal mass.

In an attempt to confirm the presence or location of the "missing" left kidney, a technetium-99m mercapto-acetyl-triglycine diuretic renogram (Fig. 2) was performed. Two focal areas of tracer concentration corresponding to 2 focal areas of functional renal tissue were noted. The left kidney was noted in an ectopic thoracic location. Prompt and adequate flow was noted in both kidneys. Split renal differential function tests revealed that the left thoracic kidney contributed to $50.2 \%$ of the overall renal function. 


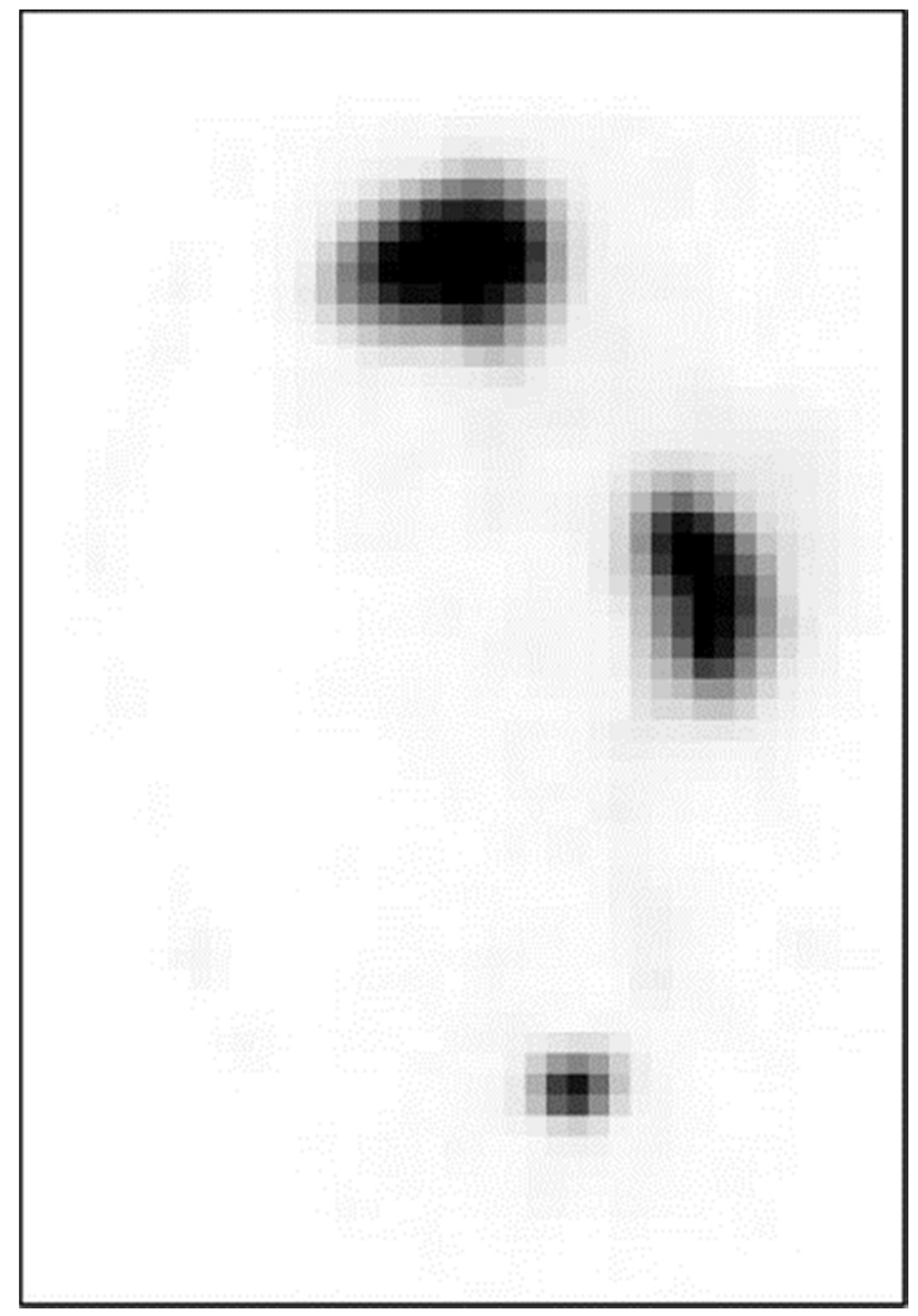

Figure 2. Technetium-99m mercapto-acetyl-triglycine renogram (posterior view) demonstrating 2 nonobstructed functional kidneys, with left kidney visualized in left thoracic region.

A noncontrast-enhanced abdominal computed tomography scan revealed the leftsided malrotated thoracic kidney (Fig. 3), without any visible calculi or other significant intra-abdominal pathologic features. The remainder of the left hemidiaphragm seemed intact. 


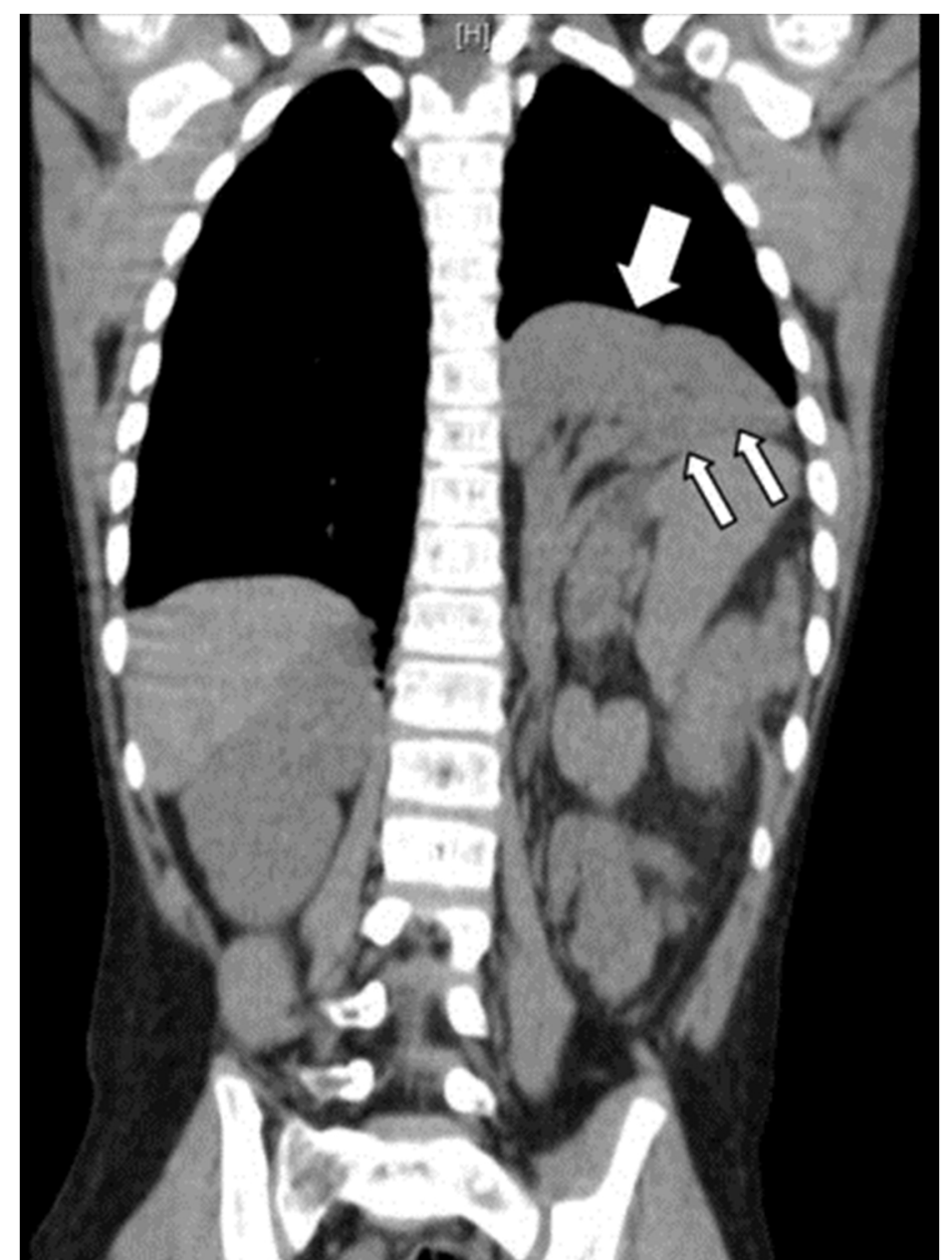

Figure 3. Computed tomography scan (coronal section) showing supradiaphragmatic, left-sided,

malrotated thoracic kidney (single arrow). Diaphragmatic outline (double arrows) is demarcated.

Despite numerous attempts at accessing the patient's perinatal records, the operative report from infancy could not be retrieved from the referring institute. However, the collateral history from the surgical and medical staff and family members did not report the presence of a congenital thoracic kidney. Additional information that excluded the possibility of a "missed" congenital ectopic thoracic kidney was the presence of normal diaphragmatic shadows (without any left mediastinal mass) on the previous series of chest radiographs, taken by the referring center, before the present admission.

Surgical repair was performed for the recurrent herniation. During this exploration, the presence of the previous dehisced diaphragmatic scar was noted, with extension of the previous repair. Bowel contents were also seen entering the thoracic cavity. The kidney was placed back in the abdomen before formal diaphragmatic closure.

The postoperative chest radiograph revealed a normal diaphragmatic outline. His surgical recovery was uneventful. 


\section{Comment}

Since the first report of the thoracic kidney in $1930,{ }^{2}$ it has remained the rarest ectopic site, with a frequency of $<5 \%$ of all the ectopic sites. ${ }^{3}$

The Bochdalek hernia is the most common type of congenital diaphragmatic hernia and is defined by a posterolateral diaphragmatic defect that allows abdominal viscera to herniate into the thoracic cavity. ${ }^{4}$ The incidence of the thoracic kidney associated with a congenital diaphragmatic hernia has been reported to be $<0.25 \%{ }^{3}$

Pfister-Goedeke and Burnier ${ }^{5}$ have classified the thoracic kidney into 4 distinct groups, according to its association: closed diaphragm; eventration of the diaphragm; diaphragmatic hernia (congenital or acquired); and traumatic diaphragmatic rupture.

Patients with a thoracic kidney can be asymptomatic throughout life or can present with dyspnea, flank pain, intermittent cough, and, even, a foul-smelling expectorate. ${ }^{6}$, ${ }^{7}$ Embryologic theories such as a pleuroperitoneal fusion abnormality or the presence of delayed mesonephric involution leading to a higher level of kidney migration could explain the occurrence of the congenital thoracic kidney. ${ }^{8}$

The differential diagnosis of a posterior mediastinal mass includes neurogenic tumor, mediastinal lymphadenopathy, descending aortic aneurysm, paraspinal abscess, and a range of pathologic esophageal processes. ${ }^{6}$

Ultrasonography has been recommended to better localize the ectopic kidney, because it lacks ionizing radiation and does not need sedation or intravenous contrast. An inexperienced ultrasonographer and excessive breathing movements can make the thoracic kidney difficult to locate using ultrasonography (as illustrated by our case). ${ }^{9}$ A renogram study could prove beneficial in these subsets of patients.

A computed tomography scan was performed in our index case, because magnetic resonance imaging (MRI) scanning was not available at the referring institution. The use of MRI-urography has been shown to be a reliable tool in the assessment of renal and ureteral anatomy of associated ectopic renal buds. ${ }^{10}$ Riccabona et al ${ }^{10}$ have even suggested the use of MRI-urography as the next investigation of choice should the ultrasound findings leave questions unanswered. Another benefit of MRI compared with computed tomography is the absence of ionizing radiation, an important factor that should be considered in imaging the pediatric population. The feasibility of MRI-urography has been confirmed in better defining anomalous renal anatomy in neonates and infants. ${ }^{10}$ The use of fetal MRI has also proved to be a useful adjunct to ultrasonography in screening for renal anomalies during pregnancy. Its role is even more relevant in the presence of oligohydramnios, in which ultrasonography could have limitations. ${ }^{11}$

Murphy et $\mathrm{al}^{1}$ reported on their experience with the largest pediatric series of the thoracic kidney and have advocated conservative management in the absence of intrathoracic bowel herniation. The standard diaphragmatic repair is, however, still recommended, with nephropexy, for the patient with intrathoracic bowel herniation. ${ }^{1}$ 
Although Murphy et al ${ }^{1}$ discussed a treatment plan for the "congenital" thoracic kidney, not much is known regarding the success of the conservative approach in the case of the acquired thoracic kidney, especially in the pediatric age group. Thus, and because of ongoing parental concern regarding the presence of recurrent herniation, after much discussion, the decision to proceed with surgical repair was taken in the present case. Although intrathoracic bowel contents seemed absent on the imaging studies, during the repair, bowel loops were visualized herniating through the previous diaphragmatic suture line.

\section{Conclusion}

We have highlighted the possibility of a thoracic kidney in patients presenting with a posterior mediastinal mass and a history of a previous diaphragmatic hernia repair. The presence of ascites, persistent coughing, and bilateral pleural effusions could have been a precipitating factor leading to recurrent diaphragmatic herniation. During ultrasonography, one should always consider searching the "treasure chest" before labeling a kidney as "absent." In equivocal cases, a renogram study will be helpful to demonstrate functional kidney parenchyma and could reveal the location of an "absent" kidney.

\section{Acknowledgment}

The authors wish to thank Professor Suzanne Delport (Neonatal Intensive Care Unit, Kalafong Hospital, Pretoria, South Africa) and Dr. Nausheen Khan (Department of Radiology, Kalafong Hospital, Pretoria, South Africa) for their assistance with this report.

\section{References}

1. Murphy JJ, Altit G, Zerhouni S. The intrathoracic kidney: should we fix it? J Ped Surg $2012 ; 47: 970-973$

2. Campbell MF. Renal ectopy. J Urol 1930;24:187-190.

3. Donat SM, Donat PE. Intrathoracic kidney: a case report with a review of the world literature. J Urol 1998;140:131-133.

4. Losanoff JE, Sauter ER. Congenital posterolateral diaphragmatic hernia in an adult. Hernia 2004;8:83-85.

5. Pfister-Goedeke L, Brunier E. Intrathoracic kidney in childhood with special reference to secondary renal transport in Bochdalek's hernia. Helvetica Paediatrica Acta 1979;34:345-457. 
6. Chong SL, Chao SM, et al. An unusual cause of mediastinal mass- a case report and literature review of the intrathoracic kidney. Proceedings of Singapore healthcare 2012;21:144-150.

7. Van der Spech $\mathrm{H}$, Van der Meer JW, Overbeke AJ, et al. Clinical reasoning and decision making in practice. A man with pneumonia and ill smelling watery sputum: the truth revealed after 80 years. Ned Tijdshr Geneeskd 2003;147:2509-2513.

8. Angulo JC, Lopez JI, Vilanova JR et al. Intrathoracic kidney and vertebral fusion: a model of combined misdevelopment. J Urol 1992;147:1351-1353.

9. Maduekwe E, Weinberg B, Samaan B, et al. Right thoracic kidney in a child with breathing difficulties-sonographic diagnosis. J Clin Ultrasound 2010;39:108-110.

10. Riccabona M, Ruppert-Kohlmayr E et al. Potential impact of pediatric MR urography on the imaging algorithm in patients with a functional single kidney. AJR Am J Roentgenol, 2004;183:795-800.

11. Gupta $P$, Kumar $S$, Sharma $R$ et al. The role of magnetic resonance imaging in fetal renal anomalies. Int J Gynaecol Obstet 2010;111:9-212. 